\title{
Patrón de Lectura Radiológica Digital para la Clasificación de Imágenes Compatibles con Silicosis
}

\author{
DIGITAL READING RADIATION PATTERN FOR CLASSIFICATION OF IMAGE ABOUT SILICOSIS (P0140-2013)
}

Bernardita Cubillos Munski', Alejandro García Herrera², Gustavo Contreras Tudela ${ }^{3}$, Carlos Villafranca Aravena ${ }^{4}$, Cristina López Soto ${ }^{5}$, Patricio Azócar Guerrero ${ }^{6}$, Gustavo Sánchez Espinoza7, Felipe Zorzano Sepúlveda ${ }^{8}$

1. Jefe Departamento de Medicina Ocupacional del Hospital del Trabajador (HT).

2-3. Profesor de la Escuela de Salud Pública de la Universidad de Chile.

4. Departamento de Medicina Ocupacional del HT.

5. Enfermera Universitaria.

6. Jefe Departamento de Imagenología del HT.

7. Tecnólogo Médico del Departamento de Imagenología.

8. Tecnólogo Médico del Servicio de Exámenes Laborales

\section{RESUMEN}

La silicosis es una enfermedad sin tratamiento. El programa de vigilancia de trabajadores expuestos a sílice está diseñado para detectarla lo más precozmente posible mediante una radiografía de tórax. A pesar de su rol fundamental, esta adolece de una gran variabilidad técnica y de interpretación. Para disminuir estos fenómenos, la OIT creó un sistema de clasificación ampliamente usado en Chile y el mundo. Este se basó en la radiografía análoga, la que ha sido reemplazada por el mismo set análogo, digitalizado. Si bien la OIT permite el uso de este nuevo tipo de imagen, indica que el patrón de comparación debe seguir siendo el análogo. Muchos estudios han demostrado que es mejor utilizar patrones de comparación digitales. El objetivo de este trabajo fue generar un set de radiografías digitales que representen las profusiones centrales de opacidades redondas del sistema de clasificación OIT. De un universo de 32.826 radiografías de pacientes expuestos a sílice, se obtuvo 252 radiografias, de las cuales 54 fueron informadas por 3 lectores acreditados, seleccionándose 8 casos que contaban con opacidades redondas puras de diferentes profusiones, las que se proponen como patrón digital para Chile.

(Cubillos B, García A, Contreras G, Villafranca C, López C, Azócar P, Sánchez G, Zorzano F, 2016. Patrón de Lectura Radiológica Digital para la Clasificación de Imágenes Compatibles con Silicosis. Cienc Trab. Ene-Abr; 18 [55]: 37-41).

Palabras claves: SILICOSIS, NEUMOCONIOSIS, SET DE LECTURA DIGITAL, CLASIFICACIÓN OIT.

\section{ABSTRACT}

Silicosis is a disease without treatment. The surveillance program for workers exposed to silica is designed to detect as early as possible by a chest radiograph. Despite its fundamental role, it suffers from a great technique and interpretation variability. To reduce these phenomena, the ILO created a classification system widely used in Chile and the world. This was based on analog radiography, which has been replaced by the same analog set, digitized. While the ILO permits the use of this new type of image it indicates that the pattern comparison must remain analog. Many studies have shown that it is best to use digital comparison patterns. The objective of this work was to generate a set of digital $\mathrm{x}$-rays representing the central profusion of round opacities of ILO classification system. From a universe of 32,826 X-rays of patients exposed to silica, 252 films, of which 54 were reported for 3 accredited readers, selected 8 cases that had pure round opacities of different profusions was obtained, which are proposed as digital pattern for Chile.

Key words: SILICOSIS, PNEUMOCONIOSIS, SET DIGITAL READING, ILO CLASSIFICATION.

\section{INTRODUCCIÓN}

La radiografía de tórax es el examen de pesquisa precoz de la silicosis. A pesar de su rol fundamental, esta presenta una gran variabilidad técnica y de interpretación. Para disminuir estos fenó-

Correspondencia / Correspondence:

Dra. Bernardita Cubillos Munski

Ramón Carnicer 185, Providencia, Región Metropolitana

Santiago, Chile

e-mail: bcubillos@achs.cl

Recibido: 29 de Octubre de 2015 / Aceptado: 15 de Diciembre de 2015

menos, la Organización Internacional del Trabajo (OIT) creó un sistema de clasificación basado en la radiografía análoga. El desarrollo tecnológico ha introducido un nuevo tipo de $\mathrm{Rx}$ de tórax (técnica digital) que ha desplazado a la análoga.

Recientemente la OIT permitió el uso de este nuevo tipo de imagen, indicando que el patrón de comparación que se debe seguir usando es el análogo. Sin embargo, no resulta fácil comparar una radiografía análoga con una digital, por lo que varios estudios han demostrado que es mejor utilizar patrones de comparación digitales.

El objetivo de este trabajo fue generar un set de radiografías digitales que representen las profusiones centrales de opacidades redondas del sistema de clasificación OIT, para su uso como patrón de comparación en Chile. 


\section{MARCO TEÓRICO}

\section{Importancia de la silicosis}

La silicosis pulmonar es la enfermedad profesional más antigua que se conoce. Los primeros casos descritos datan de los tiempos de Hipócrates en Grecia y Egipto, en trabajadores expuestos a polvo en minería. Posteriormente fue estudiada por Bernardo Ramazzini en el siglo XVIII.

Esta patología se produce por la inhalación de dióxido de silicio en sus formas cristalinas, comúnmente denominadas sílice. La enfermedad se puede presentar luego de exposiciones intensas por períodos de meses a unos pocos años (menos de 5 años) constituyendo lo que se conoce como "silicosis aguda", patología muy grave y con alta mortalidad. La forma de presentación más frecuente, tanto en Chile como en el mundo, es la silicosis crónica que corresponde a una granulomatosis pulmonar irreversible. En las etapas iniciales, esta enfermedad no provoca síntomas al trabajador, pero en las más avanzadas, el afectado presenta disnea progresiva que puede llegar hasta la insuficiencia respiratoria global y la muerte. ${ }^{1,2}$

La frecuencia de silicosis en la población laboral chilena es desconocida. Entre los años 2004 y 2005, el Departamento de Salud Ocupacional del Instituto de Salud Pública de Chile (ISP) efectuó un estudio exploratorio nacional sobre los niveles de sílice a que están expuestos los trabajadores de distintos rubros productivos. Se recolectó 364 muestras de aire en puestos de trabajo de 31 rubros diferentes en 132 empresas. ${ }^{2}$ Los resultados mostraron que en el 35\% de los rubros evaluados se sobrepasó el Límite Ponderado Permisible (LPP) de sílice definido en Decreto Supremo 594. En un 60\% de las muestras, las concentraciones ambientales mostraron valores iguales o superiores a la mitad del LPP. ${ }^{2}$ Utilizando el criterio de trabajador expuesto como aquel que permanece más del 30\% de la jornada laboral semanal en presencia de sílice, se estimó que el 5,4\% de la Fuerza de Trabajo Ocupada ${ }^{2-3}$ está expuesta a este compuesto. Si bien la cantidad de trabajadores expuestos -estimada en este estudio- supone un alto número de enfermos, la mayoría de los casos registrados en nuestro país provienen del sector minero ${ }^{4}$ asociado al sistema mutual. Aunque no hay datos publicados, se sospecha que existe un alto grado de subdiagnóstico de la enfermedad.

La silicosis es también un problema mundial, por lo que desde el año 2005 la Organización Mundial de la Salud (OMS) y la OIT están implementando una estrategia global tendiente a erradicar la enfermedad del planeta para el año 2030. Nuestro país ha adherido a esta iniciativa a través de los Ministerios de Salud y del Trabajo y Previsión Social en una declaración conjunta realizada el 13 de julio del año 2007. ${ }^{5}$ En respuesta a esta determinación, el Ministerio de Salud de Chile implementó el Plan Nacional para la Erradicación de la Silicosis, PLANESI, que guía el accionar de los distintos actores sociales en el marco del cumplimiento de la legislación vigente $\mathrm{y}$ en los roles institucionales de los organismos responsables. $^{5}$

\section{Prevención de la silicosis}

Erradicar la silicosis del planeta requiere que ningún trabajador se sobreexponga a sílice. Las medidas de control en la fuente generadora de sílice cristalina respirable no son siempre posibles. La protección respiratoria personal que se usa habitualmente tampoco garantiza que la sílice no alcance los alvéolos pulmonares de trabajadores que laboran en ambientes contaminados. Se requerirá mayor desarrollo tecnológico en ambas áreas para lograr la meta de erradicar la silicosis del mundo.

Ante esta realidad, el equipo de salud puede contribuir a aminorar los daños generados a los trabajadores como a la sociedad, mediante el diagnóstico precoz de la enfermedad, ya que, de ese modo, el afectado podrá ser retirado de la exposición, asegurándole al menos un número importante de años de sobrevida con calidad de vida adecuada.

\section{Diagnóstico precoz de la silicosis}

Con el objeto de mejorar la calidad del diagnóstico oportuno de la silicosis, el ISP-Chile implementó el Programa de Evaluación Externa de la Calidad de las prestaciones relacionadas con la Silicosis (PEECASI), el que, en lo pertinente a este proyecto, procura alcanzar la mejor calidad posible en el diagnóstico radiográfico de la enfermedad.

La normativa actual obliga al control periódico de los trabajadores con exposición presente o pasada a sílice. Los trabajadores activos deben evaluarse con radiografía de tórax con técnica de neumoconiosis según pautas de la OIT, cada uno a cuatro años, según el grado de exposición; aquellos con exposición pasada, deben ser evaluados con el mismo examen durante 15 años y con una periodicidad quinquenal.

La Asociación Chilena de Seguridad (ACHS) ha sido pionera en la aplicación de programas de prevención de las Neumoconiosis en Chile. Cuenta, desde la década de 1970, con el Programa de Vigilancia Médica de Enfermedad Profesional para Neumoconiosis, el cual evalúa periódicamente a los trabajadores en riesgo de enfermar, con una radiografía de tórax tomada e informada según los criterios OIT. ${ }^{6-8}$

\section{Radiografía de neumoconiosis}

Las manifestaciones radiográficas de la silicosis preceden al desarrollo de síntomas y a la pérdida de función pulmonar; por esto, la radiografía de tórax es la herramienta principal de reconocimiento, monitoreo y clasificación de la neumoconiosis. ${ }^{1}$

La interpretación de las imágenes en una radiografía de tórax está sujeta a la habilidad y experiencia del médico que la evalúa, por lo que existe una alta variabilidad entre lectores y también intralector, cuando una misma radiografía es informada en dos oportunidades distintas por el mismo médico. Para reducir esta variabilidad y codificar las anormalidades de manera simple y reproducible8, la OIT diseñó un sistema de clasificación que es ampliamente utilizado en el mundo. La creación de este sistema se remonta a mediados del siglo pasado, cuando sólo se disponía de radiografías análogas; estas corresponden a la imagen creada sobre una placa de acetato cubierta con una emulsión de plata, por el paso de rayos $\mathrm{X}$ a través del tórax. Este tipo de radiografía es de bajo costo, genera una buena calidad de imagen y tiene alta resolución espacial. ${ }^{9}$ El sistema OIT busca disminuir la variabilidad en los informes médicos mediante dos estrategias complementarias. Por una parte entrega directrices claras para que la imagen obtenida sea de alta calidad y, por otra, proporciona un conjunto de radiografías para ser usadas como patrón de comparación. Este está formado por nueve radiografías que representan las imágenes que pudieran ser compatibles con lesiones silicóticas, correspondientes a profusiones leve-moderada-grave, siendo las opacidades redondas las más frecuentes en silicosis. Estas radiografías se conocen como patrones centrales de profusión, forma y tamaño de las opacidades. El set de la OIT comprende otras 6 radiografías que 
proporcionan el patrón de comparación para las neumoconiosis del tipo asbestosis.

El avance de la tecnología introdujo hace algunos años la radiografía digital, en la que los rayos $\mathrm{X}$ forman la imagen sobre una placa fluoroscópica que luego se digitaliza (radiografía digital indirecta) o directamente sobre sensores digitales (radiografía digital directa). Las radiografías digitales tienen mayor resolución, son más fáciles de manipular computacionalmente para mejorar la calidad de la imagen, no requieren ser impresas (por lo que no se necesita el proceso de revelado de la placa) y se almacenan más eficientemente. Es por ello que en la actualidad la radiografía análoga es cada vez más escasa.

\section{El problema de la radiografía digital para la detección de silicosis}

El sistema OIT se basa en radiografías análogas y sus patrones de comparación (que son la base del sistema) son también análogos. El Instituto Nacional de Seguridad y Salud Ocupacional de los EE.UU. (NIOSH) fue el primero en permitir el uso de radiografías de tórax digitales para los programas de pesquisa de neumoconiosis en ese país. En ausencia de patrones digitales, NIOSH propuso utilizar las imágenes digitalizadas de los patrones análogos, como estándar de comparación. Seguidamente, la OIT adoptó igual postura.

En 2012, el ISP-Chile validó el uso de la radiografía digital con fines de pesquisa de neumoconiosis, por lo que paulatinamente se ha ido incorporando a los programas de vigilancia de trabajadores expuestos a sílice, pero su uso para fines médico-legales ha sido resistido por la falta de familiaridad de los médicos con este nuevo tipo de radiografía. Es un obstáculo a este proceso, el que se deba comparar una radiografía digital con un patrón análogo digitalizado. Por otro lado no existía un patrón digital de comparación. Cuando una imagen análoga se transforma a formato digital, se pierde información, ya que no representa fielmente el contraste ni densidad óptica obtenida en la radiografía original, lo que puede inducir a generar diagnósticos falsos negativos y/o falsos positivos. Si bien se ha descrito que existe una alta concordancia entre la interpretación de la lectura digital directa y la análoga, otros han puesto en evidencia una mayor dispersión de resultados. ${ }^{10} \mathrm{De}$ hecho, y debido a los diferentes métodos de examinación y de procesamiento de imágenes, ${ }^{11}$ la radiografía digital tiene una sensibilidad mayor que su par análogo, por lo que ofrece al médico imágenes que no se ven en la radiografía tradicional, aumentando la posibilidad de error en médicos no entrenados.

El presente trabajo propone conformar un set de radiografías digitales que constituyan el patrón de referencia para la lectura de ese tipo de imágenes obtenidas en trabajadores expuestos a sílice. Se trata, por tanto, de un proyecto de innovación tecnológica que generará un producto de aplicación inmediata y permanente. El patrón digital resultante de este proyecto podrá ser utilizado tanto en los programas de vigilancia médica de la Asociación Chilena de Seguridad, como por otros grupos interesados en el estudio y prevención de esta enfermedad.

\section{OBJETIVOS DE LA INVESTIGACIÓN}

\section{Objetivo general}

Obtener patrones radiográficos digitales para las profusiones centrales de opacidades redondas equivalentes a las actualmente en uso en versión análoga.

\section{Objetivos específicos}

1. Identificar trabajadores afectados por silicosis pulmonar cuyas radiografías representen las profusiones centrales para las opacidades redondas de la actual versión de patrones estándar de la OIT.

2. Verificar, mediante tomografía axial computada de tórax, que estos trabajadores padecen efectivamente de silicosis pulmonar y realizar el diagnóstico diferencial con otras enfermedades intersticiales nodulares.

3. Obtener de estos trabajadores la radiografía digital que constituirá el estándar digital respectivo.

4. Proponer a las autoridades nacionales el uso de estas radiografías como los patrones estándar para radiografías digitales en Chile.

\section{MÉTODO}

El proceso de selección de pacientes comenzó con la revisión de la base de datos de 30.515 informes radiográficos emitidos en Medicina del Trabajo del Departamento de Medicina Ocupacional del Hospital del Trabajador, entre enero de 2010 a diciembre de 2013, y de la base de datos de uno de los investigadores para el mismo período, correspondiente a 2.311. Entre ellos se seleccionaron 252 radiografías que presentaban las profusiones centrales $(1 / 1 ; 2 / 2 ; 3 / 3)$ de opacidades redondas.

Se revisaron las radiografías disponibles que corresponden a 54 (algunas de las placas habian sido retornadas a la ciudad de origen) para identificar aquellas de buena calidad (calidad 1 y 2 , según 0IT).7 De este modo, se obtuvo un subconjunto de radiografías que fueron informadas en forma separada por médicos certificados por el ISP. Estos informes fueron comparados para identificar aquellos concordantes, entendiéndose aquello como lecturas que no diferían más de una subcategoría en la escala de 12 puntos, descrita en la "Guía para el uso de la clasificación internacional de la OIT de radiografías de neumoconiosis".

El grupo de radiografías con informes concordantes fue revisado nuevamente por los médicos certificados, pero esta vez en la modalidad "panel”, es decir los tres simultáneamente, para identificar aquellas en las que existía unanimidad respecto del tipo de opacidad predominante y de la profusión, correspondiendo estas a 8 radiografías.

A continuación se invitó personal o telefónicamente a los 8 pacientes a colaborar en el estudio. La novena profusión, que corresponde a $3 / 3 \mathrm{p} / \mathrm{p}$, no se encontró en esta base de datos. Quienes aceptaron la invitación firmaron el consentimiento informado autorizando a que se les tomara una nueva radiografía análoga, una digital y un TAC de tórax, si es que no contaban con él.

Se obtuvo una nueva radiografía análoga que fue leída en conjunto (forma panel) para certificar que la silicosis se había mantenido en el grado informado previamente, ya que la radiografía original pudo tener más de 2 años de antigüedad. Estas se tomaron de acuerdo a las normas de la OIT (ref) con equipo Philips Optimus 50, $50 \mathrm{~kW}$ de potencia, $150 \mathrm{Kv}$ máxima, $650 \mathrm{~mA}$ máxima, capaz de generar $370 \mathrm{~mA}$ a $135 \mathrm{kVp}$, trifásico, ánodo rotatorio, colimador luminoso, foco fino $0,6 \mathrm{~mm}$ y foco grueso 1,3 $\mathrm{mm}$. La técnica radiográfica fue de $135 \mathrm{kVp}$, exposímetro automático debidamente calibrado, foco fino de $0,6 \mathrm{~mm}$. El sistema pantalla película está conformado por placas radiológicas AGFA CP-G PLUS de fabricación belga, sensibilidad verde, de baja velo- 
cidad, amplia latitud, formato $35 \times 43 \mathrm{~cm}$ y chasis radiográfico AGFA Curix, grano ultra fino (folio fine) que tiene correspondencia espectral con placa utilizada. Las imágenes latentes fueron procesadas por revelado húmedo en reveladora AGFA Classic E.O.S con parámetros de revelado debidamente controlados mediante sensitometría según protocolo ARCAL. Los químicos utilizados en el proceso corresponden a Revelador G-138 y Fijador G-134, ambos AGFA de origen belga, preparados en automixer debidamente calibrado.

La radiografía digital se obtuvo para conformar el patrón estándar con técnica directa utilizando equipo Phillips Digital Diagnost Flex con tubo SRO 33100 de $150 \mathrm{Kv}$, ánodo rotatorio y colimador luminoso y software de imágenes Flat panel. La pantalla de visualización es Barco modelo MDNG-2121, tamaño diagonal 21,3 pulgadas con resolución 1600x1200 con área de display (HxV) 432x324 mm con pixel pitch de $0,21(\mathrm{H}) \times 0,27 \mathrm{~mm}(\mathrm{~V})$ con luminiscencia de $500 \mathrm{~cd} /$ $\mathrm{m} 2$ con una dosis de radiación correspondiente a $0,1 \mathrm{mGy}$.

El TAC fue incluido en el estudio para descartar patologías de diagnóstico diferencial de la silicosis. Si el paciente contaba con un TAC, no se volvía a solicitar, considerando no exponerlos innecesariamente a radiaciones. Todos los TAC confirmaron la silicosis.

La Tomografía Axial Computada de Tórax se obtuvo en 4 pacientes que no disponían del examen previamente y su propósito fue asegurar que las opacidades vistas en las radiografías correspondían sólo a silicosis. Se utilizó protocolo de alta resolución, en equipo marca Philips, modelo Brillance, Multicorte de 64 canales, con técnica Kv 120, más / 230, grosor de corte es de $2 \mathrm{~mm}$ con un postproceso de $1 \mathrm{~mm}$, con tiempo de rotación de 0,5 segundos y con una radiación correspondiente a $671.2 \mathrm{uGm} 2$ "

No todos los pacientes concurrieron a realizarse las radiografías, faltando el paciente correspondiente al patrón $1 / 1 \mathrm{r} / \mathrm{r}$, por lo que el patrón no pudo completarse con el método propuesto. Los autores de este trabajo solicitaron al Departamento de Salud Ocupacional, de la Dirección de Políticas Públicas Saludables y Promoción del Ministerio de Salud, se les permitiera revisar su base de datos con 100 radiografías obtenidas en el 2010, con propósito similar al que motivó este estudio, para obtener las radiografías faltantes. Se leyeron en modalidad panel, obteniendo las radiografías faltantes que corresponden a la profusión $3 / 3 \mathrm{p} / \mathrm{p}$ señalada previamente y $1 / 1 \mathrm{r} / \mathrm{q}$, que fueron tomadas con técnica de digital indirecta.

Estas radiografías digitales se obtuvieron con técnica indirecta, pero de acuerdo con las normas OIT/NIOSH. Los pacientes contaban con Rx análoga y TAC de tórax contemporáneos.

La dosis de radiación total fue la mínima necesaria para obtener las imágenes requeridas por el estudio. Este procedimiento contó con la aprobación del Comité de Ética del HT.

\section{RESULTADOS}

La base de datos de Medicina del Trabajo contiene 30.515 informes para el período enero 2010 a diciembre 2013. A ello se agregan 2.311 informes provenientes de la base de datos de uno de los investigadores, lo cual hace un universo total de 32.826. De estos, se seleccionó 252 radiografías que presentaban las profusiones centrales $(1 / 1 ; 2 / 2 ; 3 / 3)$ de opacidades redondas, de las cuales 54 estaban físicamente disponibles y eran de buena calidad. Estas fueron informadas en forma separada por 3 médicos certificados por el ISP, identificándose 24 radiografías con informes concordantes (discrepancia entre lectores de no más de un grado en la escala de 12 puntos establecidos por la OIT) (Tabla 1).
Tabla 1.

Proceso de selección de radiografías análogas representativas. Universo total:

- Universo de informes base de datos Medicina del Trabajo 2010-2013

- Universo informes base datos investigador 2010-2013

Total radiografías con profusiones centrales

Total radiografias con profusión centrales físicamente disponibles

Radiografias con informes concordantes entre lectores ISP y B Reader

24

Tabla 2.

Resultados proceso de lectura panel.

Radiografias que representan patrones puros

Patrones encontrados

8

$1 / 1 p / p ; 2 / 2 p / p ; 1 / 1 q / q, 2 / 2$

Patrón no encontrado

$q / q, 3 / 3 q / q ; 1 / 1 r / r ; 2 / 2 r / r, 3 / 3 r / r$ $3 / 3 \mathrm{p} / \mathrm{p}$

Las 24 radiografías fueron revisadas nuevamente por los médicos certificados, pero esta vez en la modalidad "panel”, alcanzando consenso en 8 radiografías que representaban las profusiones centrales (1/1 p/p. 2/2 p/p, 1/1 q/q, 2/2 q/q, 3/3 q/q, 1/1 r/r; 2/2 r/r/ y $3 / 3$ r/r.) (Tabla 2).

Los ocho pacientes fueron invitados a colaborar, pero uno de ellos no se presentó -el que correspondía a la profusión $1 / 1 \mathrm{r} / \mathrm{r}-$, por lo que debió recurrirse a la base de radiografías del Departamento de Salud Ocupacional, de la Dirección de Políticas Públicas Saludables y Promoción del Ministerio de Salud, desde donde se obtuvo las dos radiografías faltantes $(3 / 3$ p/p y $1 / 1 \mathrm{r} / \mathrm{r})$.

La profusión $3 / 3 \mathrm{p} / \mathrm{p}$ que se obtuvo es central y la $\mathrm{Rx}$ de $\mathrm{r} / \mathrm{r}$ que se obtuvo es de profusión central, pero de patrón mixto (1/1 r/q). Se decidió no incluir esta radiografía dado que su interpretación pudiese generar confusión.

Se logró un patrón digital de radiografías de profusión central para 8 de los 9 patrones puros de distintos tamaños de opacidades redondas, probablemente compatibles con silicosis.

Estas imágenes ya se propusieron a las autoridades nacionales como patrones estándar para lectura de radiografías digitales de neumoconiosis en Chile.

\section{DISCUSIÓN}

Aun cuando la radiografía análoga y la digital muestran las estructuras del tórax al ser atravesadas por rayos $\mathrm{X}$, el que una genere la imagen activando una emulsión de plata y la otra un sensor digital, provocan que las imágenes no sean exactamente iguales. De hecho, la radiografía digital ofrece una calidad de imagen superior con más cantidad de detalle que, en última instancia, generan mayor incertidumbre en el médico que informa la radiografía cuando se busca en ella alteraciones sutiles como las generadas por la silicosis inicial. Existe, por tanto, una tendencia a sobrediagnosticar opacidades nodulares intersticiales en la radiografía de tórax con técnica digital. Este fenómeno ha llevado a otros grupos médicos a generar patrones de comparación digitales, los que han puesto a disposición de sus respectivos países, pero que muestran patrones mixtos.

Este estudio ha permitido identificar un grupo de radiografías homologables a aquellas del patrón analógico del sistema OIT para la lectura de la silicosis, pero al igual que ocurre con este, las radiografías propuestas en este estudio pudieran ser reemplazadas en el futuro por otras de mejor calidad. 
En efecto, las radiografías patrón aportadas por la OIT presentan algunos problemas que ponen de manifiesto la dificultad que existe para encontrar radiografías puras en la vida real. Por ejemplo, la radiografía $1 / 1 \mathrm{p} / \mathrm{p}$ del set OIT muestra un síndrome de Kaplan, es decir, silicosis más nódulos reumatoides. Esta radiografía "estándar" no corresponde a una silicosis pura, sino a una complicada por una segunda patología, lo que le resta "pureza" y, en la práctica, dificulta su uso. Las opacidades " $p$ " cuyo diámetro es menor a 1,5 mm, son poco frecuentes, en especial en la minería chilena. Aun cuando no se dispone de estadísticas oficiales, es conocido el hecho de que en Chile las opacidades más frecuentes son de tamaño "q" (1,5 a $3 \mathrm{~mm})$, seguidas por las "r" (3 a $10 \mathrm{~mm})$. En consecuencia, las radiografías que más costó obtener fueron las representantes de las opacidades "p", seguidas por las " $\mathrm{r}$ "; en cambio, las representativas de las opacidades " $q$ " fueron las primeras en ser encontradas. Otro fenómeno que está presente en las radiografías patrón OIT es que aun cuando la radiografía ha sido catalogada como representando a un tamaño y profusión particular, el análisis detallado muestra que existen opacidades de otros tamaños y que la profusión no es igual en todas las zonas afectadas. Por ejemplo, la radiografía $1 / 1 \mathrm{p} / \mathrm{p}$, tiene una región en el tercio medio derecho con opacidades " $q$ "; la radiografía $1 / 1$ q/q tiene esa profusión en el tercio superior derecho pero no en el izquierdo, donde las opacidades se presentan en cantidad menor $(1 / 0)$. Estos fenómenos ponen de manifiesto que las neumoconiosis son enfermedades evolutivas que presentan diferentes grados de desarrollo en las distintas zonas del pulmón. Fenómenos similares se pueden observar en las radiografías propuestas por este estudio. A pesar de lo ya señalado, creemos que las radiografías seleccionadas ayudarán a los médicos a generar informes más precisos, mejorando la calidad de los programas de vigilancia y del diagnóstico médico legal.

El proceso de obtención de las radiografías digitales representativas obtenidas en este estudio no estuvo exento de problemas prácticos, como por ejemplo la dificultad para que el trabajador concurriera a la toma de las radiografías. El proceso de contar con radiografías estándares debe ser continuo, porque en la medida que más imágenes vayan siendo evaluadas por un grupo mayor de médicos, se logrará una mejor representatividad de las seleccionadas.

\section{CONCLUSIONES}

El trabajo permitió obtener un grupo de ocho radiografías que representan adecuadamente las profusiones centrales de las opacidades redondas pequeñas, propias de las silicosis, equivalentes a aquellas del patrón análogo de opacidades redondas OIT. Se espera contribuir a un uso más seguro de la clasificación OIT al usar radiografías digitales por todos los médicos de este u otro país que decida adoptarlo como estándar de comparación.
1. Martínez C, Mosquera JA. Silicosis y neumoconiosis de los mineros del carbón. En: Martínez C. Manual de Neumología Ocupacional. Madrid: Ergon; 2007. pág. 197.

2. Colombia. Ministerio de la Protección Social. Guía de Atención Integral Basada en la Evidencia para Neumoconiosis (silicosis, neumoconiosis del minero de carbón y asbestosis). Bogotá: Ministerio de Protección Social; 2007.

3. Alcaíno J, Solís R, Quintanilla P. Estudio de la exposición al sílice 20042005. Santiago: ISP; 2005 [consultado dic 2015].Disponible en: http://silicosis.ispch.cl/Estudio_Exposicion_Silice_Chile.pdf

4. Contreras G. Silicosis en Chile. Cienc trab. 2004;6(11):14-18.

5. Plan Nacional de Erradicación de la Silicosis (PLANESI) 2009-2030 [pág. en Internet]. Santiago: MINSAL-MINTRAB; 2007 [citado 27 mar 2013]. Disponible en: http://www.planerradicacionsilicosis.net/

6. Chile. Ministerio de Trabajo y Previsión Social. Ley 16.744. Establece Normas sobre accidentes del trabajo y enfermedades profesionales. Santiago: MINTRAB; 1968.

7. Asociación Chilena de Seguridad (ACHS). Programa de Vigilancia Médica de Neumoconiosis. Santiago: ACHS; 1997.

8. Instituto de Salud Pública (ISP). Guia para la lectura de imágenes radiográficas de tórax análogas y digitales según normas OIT; Versión 1.0. Santiago: Ministerio de Salud; 2012. (Documento de Referencia).
9. Organización Internacional del Trabajo (OIT). Guía para el uso de la Clasificación Internacional de la OIT de Radiografías de Neumoconiosis. Ginebra: OIT; 2011. (Seguridad y Salud en el Trabajo, 22).

10. Takashinma $Y$, Suganuma $N$, Sakurazawa $H$, Itoh $H$, Hirano $H$, Shida $H$, Kusaka Y. A flat-panel detector digital radiography and a storage phosphor computed radiography: screening for pneumoconioses. J Occup Health. 2007; 49(1):39-45.

11. Won-Jeaong L, Byung-Soon Ch, Sung Jun K, Choog-Ki P, Jai-Soung P, Seok Tae, Hering KG. Development of Standard Digital Images for Pneumoconiosis. J Korean Med Sci. 2011: 26(11):1403-1408.

\section{Referencias complementarias:}

Schaefer-Prokop C, Neitzel U, Venema HW, Uffmann M, Prokop M. Digital chest radiography: an update on modern technology, dose containment and control of image quality. Eur Radiol. 2008 Sep;18(9):1818-30.

Franzblau A, Kazerooni EA, Sen A, Goodsitt MM, Lee SY, Rosenman KD, Lockey JE, Meyer CA, Gillespie BW, Petsonk El, Wang ML. Comparison of Digital Radiographs with Film Radiographs for the Classification of Pneumoconiosis. Acad Radiol. 2009;16(6):669-677. 